of both CFSC-2G and isolated HSC was inhibited dose-dependently by EMD409915, with complete abrogation of migration at 10-6M. There was no effect of beta 3 integrin inhibition on cell proliferation in both cell lines. Pre-incubation of the cells with the beta 3 integrin inhibitor completely blocked PDGF-stimulated phosphorylation of p38 MAPK and FAK. Conclusion: PDGF-BB-induced HSC migration can be completely blocked in vitro by the beta 3-integrin specific non-peptidic inhibitor EMD409915. Specific integrin inhibition provides a novel promising strategy for antifibrotic therapy.

\section{DIFFERENTIAL EXPRESSION OF TWO ISOFORMS OF THE CHEMOKINE RECEPTOR CXCR3 DETERMINES THE ULTIMATE EFFECT OF IP-10 ON SURVIVAL OF HUMAN} HEPATIC STELLATE CELLS (HSC)

I. Petrai $^{1}$, A. Bonacchi ${ }^{1}$, P. Romagnani ${ }^{2}$, M. Parola ${ }^{3}$, B. Mazzinghi ${ }^{2}$, M. Rotondi ${ }^{2}$, P. Failli ${ }^{4}$, S. Aleffi ${ }^{1}$, C. Bertolani ${ }^{1}$, E. Novo ${ }^{3}$, E. Zamara ${ }^{3}$, M. Pinzani ${ }^{1}$, G. Laffi ${ }^{1}$, P. Gentilini ${ }^{1}$, F. Marra ${ }^{1} .{ }^{1}$ Dipartimento Di Medicina Interna, University of Florence, Florence, Italy; ${ }^{2}$ Dipartimento Di Fisiopatologia Clinica, University of Florence, Florence, Italy; ${ }^{3}$ Dipartimento Di Medicina E Oncologia Sperimentale, University of Turin, Turin, Italy; ${ }^{4}$ Dipartimento Di Farmacologia Preclinica E Clinica, University of Florence, Florence, Italy

Activated human HSC express the chemokine receptor CXCR3, which binds IP-10 and induces cell migration. Recently, an alternatively spliced isoform, CXCR3b, has been described in microvascular endothelial cells. Unlike the "classic" isoform, CXCR3a, CXCR3b leads to growth inhibition and apoptosis, and mediates angiostatic effects of IP-10. In HSC, the possible role of CXCR3b expression is unknown. In this study we investigated the biologic significance of the IP-10/CXCR3 system in HSC, considering the role of CXCR3b. Apoptosis of activated HSC was induced by Fas activation and/or protein synthesis inhibition by cycloheximide, and was evaluated with PARP cleavage (western blotting) and caspase 3 activation (fluorometric assay). IP-10 secretion by HSC was measured by ELISA. IP-10 was detectable in HSC supernatants, and increased in response to IFN-gamma. Activating anti-Fas antibody and/or cycloheximide induced HSC apoptosis, which was inhibited by low concentrations $(20 \mathrm{ng} / \mathrm{ml})$ of IP-10. However, the inhibition of apoptosis by IP-10 was variable in different cell lines and tended to disappear after prolonged cell culture, when a pro-apoptotic effect was actually observed. To test for this apparent paradox, expression of CXCR3a and CXCR3b was evaluated in cells that did not show increased survival with IP-10. Surprisingly, these cells expressed much higher levels of CXCR3b than CXCR3a, thus providing a mechanism for the reversal of the anti-apoptotic effects by IP-10. We conclude that HSC may express both CXCR3 isoforms, that mediate opposing effects of IP-10 on apoptosis. The ultimate biologic effect of autocrine IP-10 secretion depends on the relative expression of the two isoforms.

\section{HUMAN STEM CELLS CAN REGENERATE AND TRANSDIFFERANTIATE IN A IMMUNOCOMPETENT RAT MODEL OF ACUTE HEPATITIS}

A.C. Piscaglia ${ }^{1}$, C. Di Campli ${ }^{1}$, M. Nestola ${ }^{1}$, S. Rutella ${ }^{2}$, G. Bonanno ${ }^{2}$, A. Mariotti ${ }^{2}$, G. Zannoni ${ }^{3}$, F.M. Vecchio ${ }^{3}$, P. Pola ${ }^{4}$, G. Leone ${ }^{2}$, G. Gasbarrini ${ }^{1}$, A. Gasbarrini ${ }^{4} .{ }^{1}$ Internal Medicine, Catholic Univeristy, Gemelli Hospital, Rome, Italy; ${ }^{2}$ Haematology, Catholic Univeristy, Gemelli Hospital, Rome, Italy; ${ }^{3}$ Pathologic Anatomy, Catholic Univeristy, Gemelli Hospital, Rome, Italy; ${ }^{4}$ Medical Pathology, Catholic Univeristy, Gemelli Hospital, Rome, Italy

Background and aim: Tissue homeostasis and turnover are guaranted by the stem proliferating reserve. Several studies, performed with immunodeficient animals, suggested that a particular degree of plasticity is shown by the haematopoietic stem cell (HSC) compartment, that could be a source for liver regeneration. We aimed to explore the hepatic differentiation po- tential of human HSC also in immunocompetent rats after a toxic liver damage.

Materials and methods: Winstar rats were so sorted: Group A: liver damage with Allyl Alcohol (AA) intraperitoneally (ip) injection and killing at 2, 4, 7 days after. Group B: human HSC injection (ip) and killing at 1, 3, 6 days after. Group C: liver damage (AA, ip); human HSC injection (ip) after 1 day and killing at 1, 3, 6 days after HSC injection. Group D: sacrifice without any treatment. Livers, spleens and bone marrows were analysed for human HSC detection using Flow Cytometry (FCM); livers were also tested with immunohistochemistry (IHC) to demonstrate HSC transdifferentiation into hepatocytes.

Results: FCM confirmed the presence of human HSC (groups B and C) and revealed their selective recruitment into the damaged livers (group C) in comparison with the controls (group B). IHC demonstrated the HSC capability to transdifferentiate into hepatic cells.

Conclusions: Our study demonstrated that HSC are able to contribute in liver regeneration after an acute toxic damage in not-myeloablated recipients, opening doors to speculations about their future applications for human liver diseases, without the need of immunosuppressive pre-treatments.

\section{STIMULATION OF INTERSTITIAL COLLAGENASE ACTIVITY BY HALOFUGINONE IN HEPATIC STELLATE CELLS IS DEPENDENT ON ACTIVATION OF P38 AND NF KAPPA B}

$\underline{\text { Y. Popov }}^{1}$, E. Patsenker ${ }^{1}$, E. Niedobitek ${ }^{1}$, M. Bauer ${ }^{1}$, M. Pines ${ }^{2}$, D. Schuppan ${ }^{1} .{ }^{1}$ Dept. of Medicine I, University of Erlangen, Erlangen, Germany; ${ }^{2}$ Institute For Animal Science, Bet Dagan, Israel

Background: The semisynthetic plant alkaloid Halofuginone (HAL) was reported to ameliorate rat liver fibrosis. We recently found that the antifibrotic potential of HAL is likely to be associated with a strong MMP induction in activated hepatic stellate cells (HSCs). Our present study aimed to investigate molecular mechanisms involved in this effect.

Methods: The HSC line CFSC-2G and isolated rat HSCs were incubated with increasing concentrations of HAL. mRNA steady state levels of MMP$3,-13$ were quantified by real-time PCR. Interstitial collagenase activities were assessed in conditioned media. p38 MAPK and nuclear factor kappa B (NF kappa B) pathways were evaluated by western blotting (WB) of cell lysates and nuclear extracts with phospho-p38 and p65 NF kappa B antibodies, respectively. SB 203580 and MG132 were used to block p38 and NF kappa B signaling.

Results: Interstitial collagenase activity of HSC cultures increased doseand time-dependently up to 3-fold in the presence of HAL at 20-200 nM. At 200 nM HAL rapidly up-regulated MMP-3 and MMP-13 (interstitial collagenase) mRNA expression 10-50-fold in CFSC-2G and HSC, which was dose-dependently inhibited by pre-incubation of cells with both SB 203580 and MG132 prior to treatment with HAL. Activation of p38 and NF kappa B pathways was confirmed by WB in HSC treated with HAL for 0,5-6 hours.

Conclusions: 1. HAL stimulates interstitial collagenolytic activity of HSC in a hitherto unmatched way via activation of $\mathrm{p} 38$ MAPK and NF kappa B pathways; 2 . HAL is a highly promising drug for combination therapy of liver fibrosis.

\section{FLUORESCENCE RESONANCE ENERGY TRANSFER (FRET) BETWEEN TIMP-1 AND MMP-9 REVEALED INTRACELLULAR COMPLEX FORMATION IN LIVER CELLS}

M. Roderfeld ${ }^{1}$, B. Giese ${ }^{2}$, G. Muller-Neven ${ }^{2}$, B. Jansen ${ }^{1}$, S. Matern ${ }^{1}$, E. Roeb ${ }^{1} .{ }^{I}$ Clinic For Medicine III, University Hospital Aachen, Aachen, Germany; ${ }^{2}$ Dept. of Biochemistry, University Hospital Aachen, Aachen, Germany

Background: Expression of tissue inhibitor of metalloproteinases-1 (TIMP-1) is closely correlated with liver fibrogenesis. In the context of TIMP-1-antagonisation by matrix metalloproteinases (MMPs) we inves- 